\title{
Efficacy and Tolerability of Quetiapine Extended Release (XR) as Monotherapy in the Treatment of Major Depressive Disorder
}

\author{
Ozden Arisoy, MD ${ }^{1,2}$, Michelle Murphy, $M D^{3}$, Edwin Meresh, MD ${ }^{1}$ and Angelos Halaris, MD, PhD ${ }^{1^{*}}$ \\ ${ }^{1}$ Department of Psychiatry and Behavioral Neurosciences, Loyola University Stritch School of Medicine, USA \\ ${ }^{2}$ Department of Psychiatry, Bolu Abant Izzet Baysal University, Turkey \\ ${ }^{3}$ Department of Psychiatry, Advocate Lutheran General Hospital, USA
}

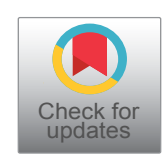

*Corresponding author: Angelos Halaris, MD, PhD, Professor of Psychiatry, Department of Psychiatry and Behavioral Neuroscience, Loyola University Stritch School of Medicine, 2160 South First Avenue, Maywood, IL 60153, USA, Tel: 708216-3752, Fax: 708-216-6840

\begin{abstract}
The aim of this study was to confirm the efficacy and tolerability of Quetiapine XR as monotherapy in the treatment of Major Depressive Disorder (MDD) and determine the optimal dosing regimen to maximize efficacy and reduce non-compliance due to side effects. This was a 12-week study with MDD subjects. The primary outcome measure was the Hamilton Rating Scale for Depression (HAMD) total score comparing baseline to end of treatment at week 12. Other assessments included the Hamilton Rating Scale for Anxiety (HAMA), Beck Depression Inventory (BDI), Beck Anxiety Inventory (BAI), and Perceived Stress Scale (PSS). Patients were flexibly dosed with Quetiapine XR beginning at $25 \mathrm{mg} /$ day and titrating up to $300 \mathrm{mg} /$ day as necessary. Patients were evaluated at baseline, and weeks 1, 2, 4, 8, 12. Of the 47 patients who completed baseline assessments, 43 were in the Intent-to-Treat (ITT) analysis and 28 completed at least 8 weeks of the study. There was no statistical significance in the baseline HAMD, HAMA, BDI, BAI or PSS scores between non-completers and completers. By week-1, patients on Quetiapine XR had a reduction in HAMD-17 scores from 24.64 to $15.68(p<0.05)$. By week 12, the median HAMD-7, HAMD-17, and HAMD-21 scores were < 7. Overall remission rate was $64.3 \%$. Patients also experienced a significant decrease in HAMA scores from 21 (moderate anxiety) at baseline to 4 (minimal to no anxiety) at week 12. This decrease was much more pronounced in the high-anxiety group. We concluded that in patients with MDD, Quetiapine XR is an effective and relatively well tolerated monotherapy with the onset of therapeutic effect in both depression and anxiety symptoms occurring as early as week-1. We further determined that the optimal average daily dose (dose with greatest efficacy and least amount of adverse effects) is about $175 \mathrm{mg} /$ day.
\end{abstract}

\author{
Keywords \\ Quetiapine XR, Monotherapy, Major depressive disorder
}

\section{Introduction}

\section{Background}

According to the latest epidemiological data published by the World Health Organization (WHO), Major Depressive Disorder (MDD) affects more than 300 million people worldwide [1]. In the United States, MDD is the leading cause of disability for individuals ages 1544 , resulting in an economic burden that exceeds $\$ 200$ billion per year $[2,3]$. With these staggering societal costs, it is important to reconsider the efficacy and tolerability of current antidepressant treatment regimens. With over 25 agents currently approved to treat MDD, the response rates to initial antidepressant therapy are estimated around 50\% [4]. This pales in comparison to other chronic disease treatments, such as amlodipine-benazepril, a combination therapy for hypertension, which demonstrates a response rate as high as $87 \%$ [5]. Remission rates are reported to be even lower at $30 \%-40 \%$ while around two-thirds of patients will not achieve full symptomatic remission with an initial agent [6]. Furthermore, the residual depressive symptoms for the $60-70 \%$ of patients who do not experience remission are very likely associated with the increasing morbidity, mortality and the overall economic and societal burden of MDD $[7,8]$. Suicidal behavior, the most tragic

Citation: Arisoy O, Murphy M, Meresh E, Halaris A (2019) Efficacy and Tolerability of Quetiapine Extended Release (XR) as Monotherapy in the Treatment of Major Depressive Disorder. Int J Depress Anxiety 2:017. doi.org/10.23937/2643-4059/1710017

Accepted: November 23, 2019: Published: November 25, 2019

Copyright: (C) 2019 Arisoy O, et al. This is an open-access article distributed under the terms of the Creative Commons Attribution License, which permits unrestricted use, distribution, and reproduction in any medium, provided the original author and source are credited. 
consequence of untreated or unsuccessfully treated depression, commonly occurs in the first few weeks of antidepressant treatment before the onset of therapeutic action and is strongly related to certain specific symptoms of depression like anxiety, agitation and insomnia [9].

A partial explanation for the low response and remission rates in antidepressant drug therapy is late onset of response and/or poor tolerability. Unfortunately, most first line drugs take upwards of two weeks or longer to take effect [6] and a trial period of 4 to 8 weeks is generally required to determine whether an agent is likely to be efficacious for a patient [10]. For the selective serotonin reuptake inhibitor class alone, which is the most widely used first-line treatment for MDD, $27-43 \%$ of patients report discontinuing treatment due to adverse effects [11]. Both persistent early-onset side effects and late-onset side effects from first-line antidepressant treatments (e.g. fatigue, sexual dysfunction, weight gain, sleep disturbances, and cognitive impairment) can significantly impact patient adherence and overall treatment outcome [12]. Hence, there is a great need for more efficacious and better tolerated treatment options for patients with MDD. Once-daily extended release Quetiapine Fumarate (Quetiapine $\mathrm{XR}$ ), an atypical antipsychotic, is one possible option.

The purpose of this study was to expand research on the efficacy and tolerability of Quetiapine XR as monotherapy for MDD and also to determine the optimal dosing regimen (i.e. dosing that provides reliable efficacy and minimizes the side effect profile).

\section{Material and Methods}

\section{Study population}

The study was approved by the Institutional Review Board (IRB) of Loyola University Medical Center and was conducted according to the principles of the Declaration of Helsinki. The study included only those candidates capable of understanding the nature of the study and giving informed consent. Female and male patients, 20-65 years of age, diagnosed with MDD were included in the study. They were in either in their first or recurrent episode based on the Diagnostic and Statistical Manual of Mental Disorders-Fourth Edition (DSM-IV). Patients did not meet criteria for treatment resistant MDD, defined as failure to respond to at least two antidepressant drugs administered in commonly prescribed doses and for an adequate length of time. Females who were pregnant, lactating or breast feeding were excluded from the study. Patients with other DSM-IV Axis I disorders, other than MDD co-morbid with Generalized Anxiety Disorder (GAD), were excluded. Patients taking any of the following compounds were excluded from the study: Cytochrome P450 and P4503A4 in- ducers (e.g. ketoconazole, itraconazole, fluconazole, erythromycin, clarithromycin, troleandomycin, indinavir, nelfinavir, ritonavir, fluvoxamine, saquinavir, phenytoin, carbamazepine, barbiturates, rifampin, St. John's Wort, and glucocorticoids). Patients undergoing any other therapeutic interventions (e.g. psychotherapy) were excluded from the study. Patients with chronic medical illnesses (e.g. diabetes, hypertension) were excluded, if they were unstable or not adequately maintained on medication.

Flow chart of the study is presented in Figure 1.

\section{Study design}

Screening: Patients were screened with the following screening tools: Mini International Neuropsychiatric Interview (MINI) and a Family History Questionnaire. Patients receiving anti-anxiety or hypnotic medications were allowed to continue taking the medication at the discretion of the investigator. Female patients were given a urine-based pregnancy test during this screening process and at the final visit at week 12.

Baseline: After the initial screening, patients completed a baseline evaluation including: Hamilton Rating Scale for Depression and Hamilton Rating Scale for Anxiety, Beck Depression Inventory (BDI), Beck Anxiety Inventory (BAI), and Perceived Stress Scale 14 (PSS14).

Dosing regimen: After completing the initial baseline assessment scales, the participants began the following 12-week regimen with Quetiapine XR, if the dose titration was tolerated. Patients were instructed to take their dose at least one hour before bedtime and not during the daytime.

Step-1: Starting dose: $25 \mathrm{mg} /$ day until end of day 3.

Step-2: $50-100 \mathrm{mg} /$ day from day 4 until end of day 7.

Step-3: $150 \mathrm{mg} /$ day from day 8 until end of day 21 or longer; the patient was maintained at this dose of 150 $\mathrm{mg} /$ day, if the response was satisfactory.

Step-4 (only if needed): 200-300 mg/day from day 22 until end of treatment with possibility of rolling back the dose to $150 \mathrm{mg} /$ day, if not well tolerated.

\section{Efficacy tolerability measurement}

Outcome measurements of efficacy were based on the following rating scales completed at weeks one, two, four, eight, and twelve: HAM-D, HAM-A, BDI, BAI and PSS.

Tolerability measurements were made on subjective symptom reporting by the patients at each visit, and also their willingness to remain in the study.

\section{Statistical analysis}

Statistical Package for Social Sciences (SPSS) 22 (SPSS Inc., Chicago, IL) software program was used 


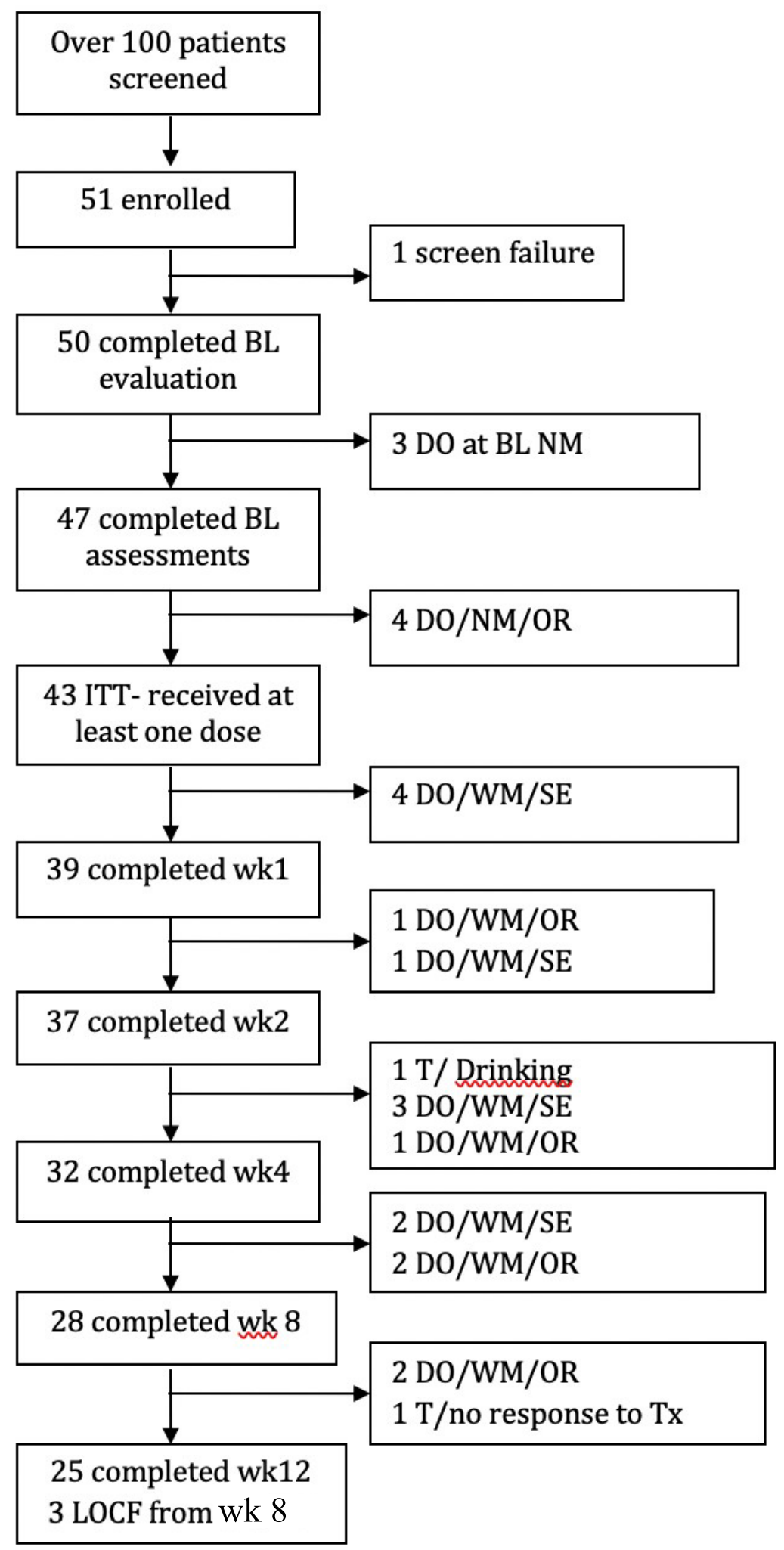

Figure 1: Flow chart of study participants.

BL: Baseline; DO: Dropout; WM: With Medication; NM: No Medication; SE: Side Effects; T: Terminated by Investigator; Tx: Treatment; OR: Other reasons included moving out of state, getting a job or having to attend to family needs and therefore being unable to meet schedule of appointments; LOCF: Last Observation Carried Forward.

for statistical analysis. Continuous data was expressed as either mean (standard deviation) or median (interquartile range) and categorical data was expressed as numbers (percentages). Normal distribution was tested by Kolmogorov-Smirnov test first and then normally distributed variables were compared with independent samples $t$ test while non-normally distributed variables were compared with the Mann Whitney $U$ test. Paired samples $t$ test or two related samples $t$ test was applied to analyze the difference between baseline and week 12 measurements according to the distribution of variables. Nonparametric 
Freidman test was applied for non-normally distributed variables among six consecutive measurements of depression and anxiety to explore the difference between baseline, $1^{\text {st }}$ week, $2^{\text {nd }}$ week, $4^{\text {th }}$ week, $8^{\text {th }}$ week and $12^{\text {th }}$ week. When a significant time effect was demonstrated in the Friedman test, the Wilcoxon signed-rank test for paired samples was used as a follow-up procedure to make post hoc pair wise comparisons. The main evaluation index of the study was the change in HAMD-17 score. The patient group was then divided into low anxiety and high anxiety groups by taking a median split of HAMA score of 22 and the effect Quetiapine had on HAMD-17 and HAMA scores in these two groups was analyzed with a mixed ANOVA repeated measures analysis. If Mauchly's test of sphericity could not be assumed, the Greenhouse-Geisser adjustment was used for the numerator and denominator degrees of freedom in the $F$ test. Post hoc multiple comparisons for observed mean between groups was performed with Tukey's test. Statistical significance was based on $p<0.05$.

\section{Results}

\section{Study participants}

Of the 100 patients that were screened via phone or in person, 51 patients were enrolled in the study. But, one patient failed screening, so 50 patients completed the baseline evaluation. Three of them dropped out at this stage and 47 patients completed the baseline assessments. Later, four of them dropped out due to other reasons like relocating to another city before receiving any medication and these 43 patients were included in our Intent-to-Treat Analysis (ITT) (i.e. those who received at least one dose of Quetiapine XR). Four ITT patients dropped out due to medication side effect (daytime sedation) and 39 of them completed the week 1 assessment. One more dropped out due to effects and another dropped out due to other reasons, so that 37 patients completed the week 2 assessments. One was terminated due to drinking, three dropped out due to side effects and one dropped out due to other reasons, so that 32 completed the week 4 assessments. Later two more dropped out due to side effects and two dropped out due to other reasons, so that 28 patients completed the week 8 assessments and those were considered to be completers (i.e. those who completed at least 8 weeks of treatment and whose last observation would be carried forward to week 12). Later on, two more dropped out due to other reasons, and one was terminated due to non-response to treatment, so that 25 of them completed the week 12 assessments. Figure 1 shows the detailed breakdown of our participant selection process and attrition throughout the study.

The mean age of our study group was $43.53 \pm 12.02$ years, $55.3 \%$ were female and $44.7 \%$ were male. In terms of ethnic background, 22 (46.8\%) of them were Caucasian and 25 (53.2\%) were non-Caucasian. Of the female patients, $83.3 \%$ were premenopausal and $16.7 \%$ were postmenopausal. There were no smokers in the patient group. The average Body Mass Index (BMI) in the study population was $31.80 \pm 6.62$, which is considered obese according to WHO [13].

Table 1: Demographics and baseline rating scales of completers vs. non-completers.

\begin{tabular}{|c|c|c|c|c|}
\hline Variable & $\begin{array}{l}\text { Completers } \\
(\mathbf{N}=\mathbf{2 8})\end{array}$ & $\begin{array}{l}\text { Non-completers } \\
(\mathrm{N}=19)\end{array}$ & $t / z / X^{2}$ & $\mathbf{p}$ \\
\hline Age (year) & $44.43(11.94)$ & $42.21(12.30)$ & $0.61^{t}$ & 0.541 \\
\hline \multicolumn{5}{|l|}{ Sex } \\
\hline Female & $13(46.4 \%)$ & $13(46.4 \%)$ & $2.21 x^{2}$ & 0.137 \\
\hline Male & $15(33.3 \%)$ & $6(53.6 \%)$ & & \\
\hline \multicolumn{5}{|l|}{ Ethnic group } \\
\hline Caucasian & $16(57.1 \%)$ & $6(57.1 \%)$ & $2.97 x^{2}$ & 0.085 \\
\hline Non-caucasian & $12(42.9 \%)$ & $16(21.4 \%)$ & & \\
\hline BL HAM D-7 & $15.61(4.63)$ & $15.63(3.21)$ & $-0.02^{t}$ & 0.984 \\
\hline BL HAMD-17 & $24.93(6.03)$ & $25.53(5.88)$ & $-0.33^{t}$ & 0.738 \\
\hline BL HAMD-21 & $26(8)$ & $27.5(7)$ & $-0.21^{z}$ & 0.828 \\
\hline BL HAMA & $23.43(9.87)$ & $20.79(7.13)$ & $1.00^{t}$ & 0.323 \\
\hline BL BDI & $25.30(9.26)$ & $27.58(10.06)$ & $-0.79^{t}$ & 0.431 \\
\hline BL BAI & $23.00(12.74)$ & $22.11(13.60)$ & $0.22^{t}$ & 0.821 \\
\hline BL PSS 14 & $49.12(7.37)$ & 48.72 (8.99) & $0.15^{t}$ & 0.875 \\
\hline BL dose & $25(25)$ & $25(25)$ & $-0.63^{z}$ & 0.529 \\
\hline
\end{tabular}

For categorical variables $\mathrm{N}(\%)$ is given and a $\mathrm{X}^{2}$ is obtained for chi square test. For continuous variables, mean (standard deviation) or median (interquartile range) is given according to the test applied and $t$ scores are obtained for student $t$ test, $z$ scores are obtained for Mann Whitney U test. Note: " $p$ < 0.05. BL: Baseline; HAMD: Hamilton Rating Scale for Depression; HAMA: Hamilton Rating Scale for Anxiety; BDI: Beck Depression Inventory; BAI: Beck Anxiety Inventory; PSS: Perceived Stress Scale. 
Current episode length was 60.74 months $(6-300$ weeks). At baseline the mean HAMD-7 score was 15.52 \pm 4.08 , the HAMD-17 score was $25.17 \pm 5.91$, and the HAMD-21 score was $27.11 \pm 6.27$, and the BDI score was $26.24 \pm 9.56$. For anxiety levels, the baseline mean HAMA score was $22.36 \pm 8.88$ and BAl score was 22.63 \pm 12.96 . Mean Baseline PSS 14 score was $48.95 \pm 7.97$.

As seen in Table 1, there was no significant difference in the demographic data and baseline rating scales of our completer group $(n=28)$ and non-completer group $(n=19)$. This indicates that those patients who discontinued the study prematurely were not significantly more ill than those who completed, which would have falsely inflated our results.

\section{Quetiapine efficacy measures}

Table 2: Efficacy measurements with rating scales baseline vs. week 12 in study group.

\begin{tabular}{|l|l|l|l|l|}
\hline Variable & $\begin{array}{l}\text { Baseline } \\
\mathbf{( N = 2 8 )}\end{array}$ & $\begin{array}{l}\text { Week 12 } \\
\mathbf{( N = 2 8 )}\end{array}$ & $\mathbf{t} / \mathbf{z}$ & $\mathbf{p}$ \\
\hline HAMD 7 & $14.5(8)$ & $3(9)$ & $-4.54^{z}$ & $0.005^{\star}$ \\
\hline HAMD 17 & $24.64(6.19)$ & $6.27(6.71)$ & $13.76^{\mathrm{t}}$ & $0.005^{\star}$ \\
\hline HAMD 21 & $26.5(13)$ & $5.5(14)$ & $-4.62^{z}$ & $0.005^{\star}$ \\
\hline HAMA & $21(14)$ & $4(8)$ & $-4.51^{z}$ & $0.005^{*}$ \\
\hline BDI & $23(15)$ & $2.5(13)$ & $-4.43^{z}$ & $0.005^{*}$ \\
\hline BAI & $19(17)$ & $5(8)$ & $-3.87^{z}$ & $0.005^{*}$ \\
\hline PSS14 & $48.83(7.79)$ & $34.74(9.46)$ & $5.17^{t}$ & $0.005^{*}$ \\
\hline
\end{tabular}

For continuous variables, mean (standard deviation) or median (interquartile range) is given according to the test applied and $t$ scores are obtained for paired $t$ test, $z$ scores are obtained for two related samples test.

Note: ${ }^{*} \mathrm{p}<0.05$. BL: Baseline; HAMD: Hamilton Rating Scale for Depression; HAMA: Hamilton Rating Scale for Anxiety; BDI: Beck Depression Inventory; BAI: Beck Anxiety Inventory; PSS: Perceived Stress Scale.
As shown in Table 2, our results demonstrate significant improvement in all efficacy measures from baseline to week $12(p<0.001)$ (Table 2). These measurements included HAMD-7, HAMD-17, HAMD-21, HAMA, BDI, BAI, and PSS14.

For the HAMD scores, all participants' baseline scores were in either the moderate or severe depression range with a mean HAMD-17 score of 24.64 and a median HAMD-21 score of 26.5. At week 12, mean and median HAMD-7, 17 and 21 were all $<7$. According to response criteria of $\geq 50 \%$ reduction of the HAMD-17 total score, and remission criteria of HAMD-17 total score of $\leq 7$ at endpoint, the response rate was $78.6 \%$ and the remission rate was $64.3 \%$ by week 12 . The response and remission rates at each time point increased steadily as can be seen in Figure 2. The BDI scores support the HAMD findings with a median drop from 23 (moderately depressed) to 2.5 (normal mood) [14].

With regard to anxiety, the HAMA results at baseline showed a median score of 21 placing these patients in the moderate anxiety range. At week 12 , the median score dropped to 4, placing these patients in the normal anxiety range. This finding was also supported by the BAI median scores which dropped from 19 (moderate anxiety) to 5 (minimal-to-no anxiety) [15]. The stress level decreased as reflected in the drop of PSS14 scores from 48.84 to $34.74(p<0.05)$.

Overall, utilizing the Friedman non-parametric statistical test, we found a highly significant effect of time on all psychiatric measures with Quetiapine treatment $(p<0.001)$. This confirms the consistent efficacy of Quetiapine XR from week 1 through week 12. In terms of onset of action; significant changes were observed as early as week 1 . Post hoc pairwise comparisons showed significant decreases from baseline to week 1 on in all HAMD-7, HAMD-17, HAMD-21, BDI scores (Figure 3A,

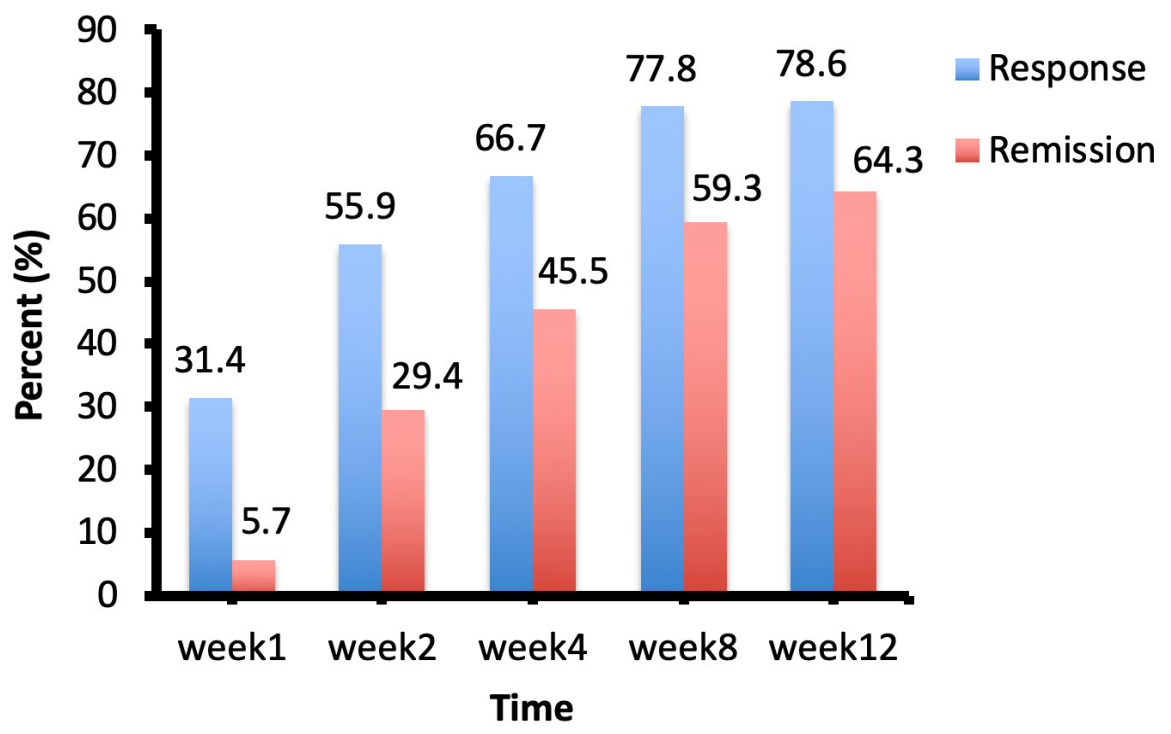

Figure 2: Response and remission rates over time. The rates are shown as percent change (\%). Response criterion was $\geq$ $50 \%$ reduction of the HAMD-17 total score and remission criterion was a HAMD-17 total score of $\leq 7$. 


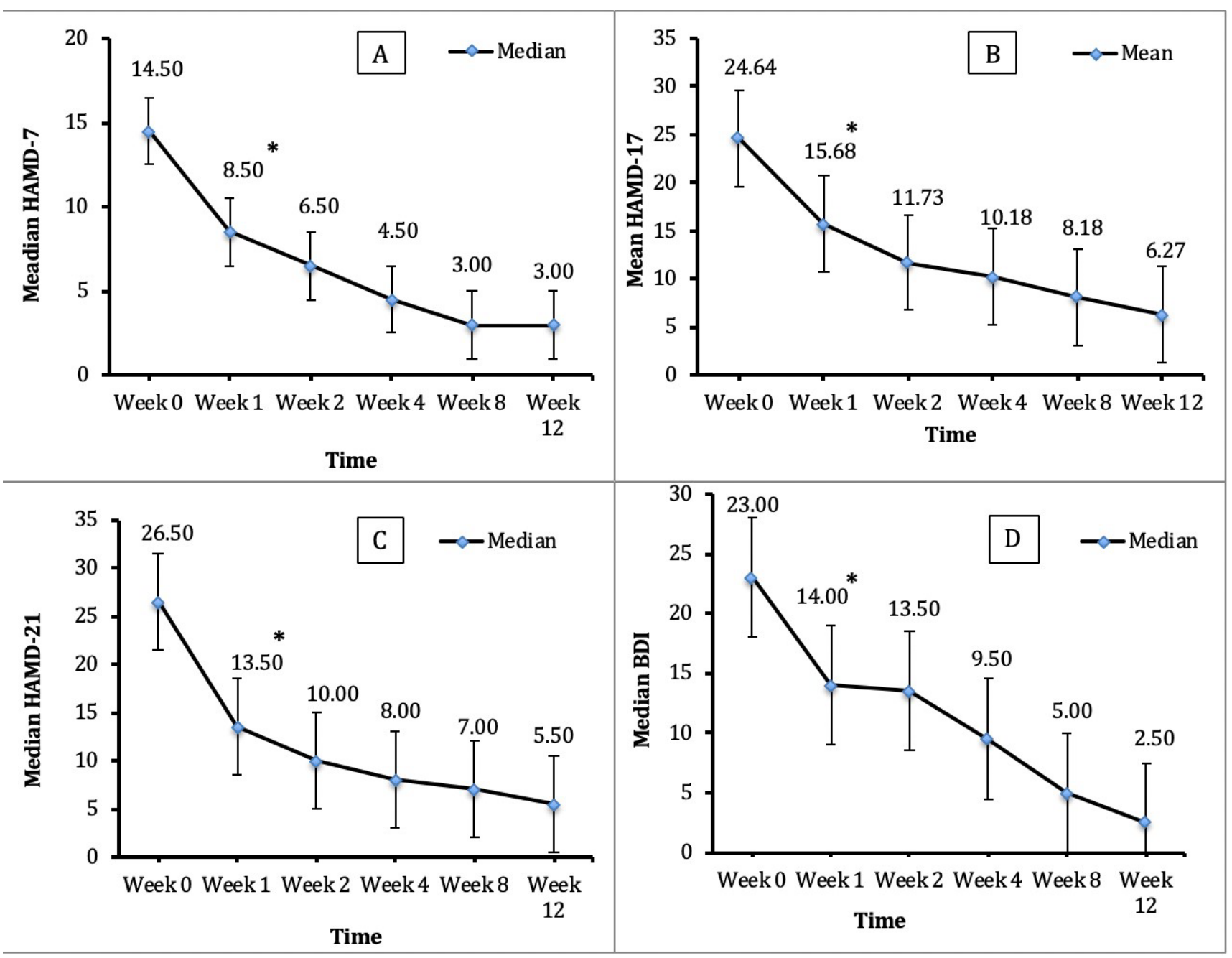

Figure 3: Changes in HAMD 7-17-21 and BDI scores from baseline through week 12. A) Change in Median HAMD-7 score; $B)$ Change in Mean HAMD-17 score; C) Change in Median HAMD-21 score; D) Change in Median BDI score. Note: ${ }^{*}<0.05$ compared to baseline.

HAMD: Hamilton Rating Scale for Depression; BDI: Beck Depression Inventory.

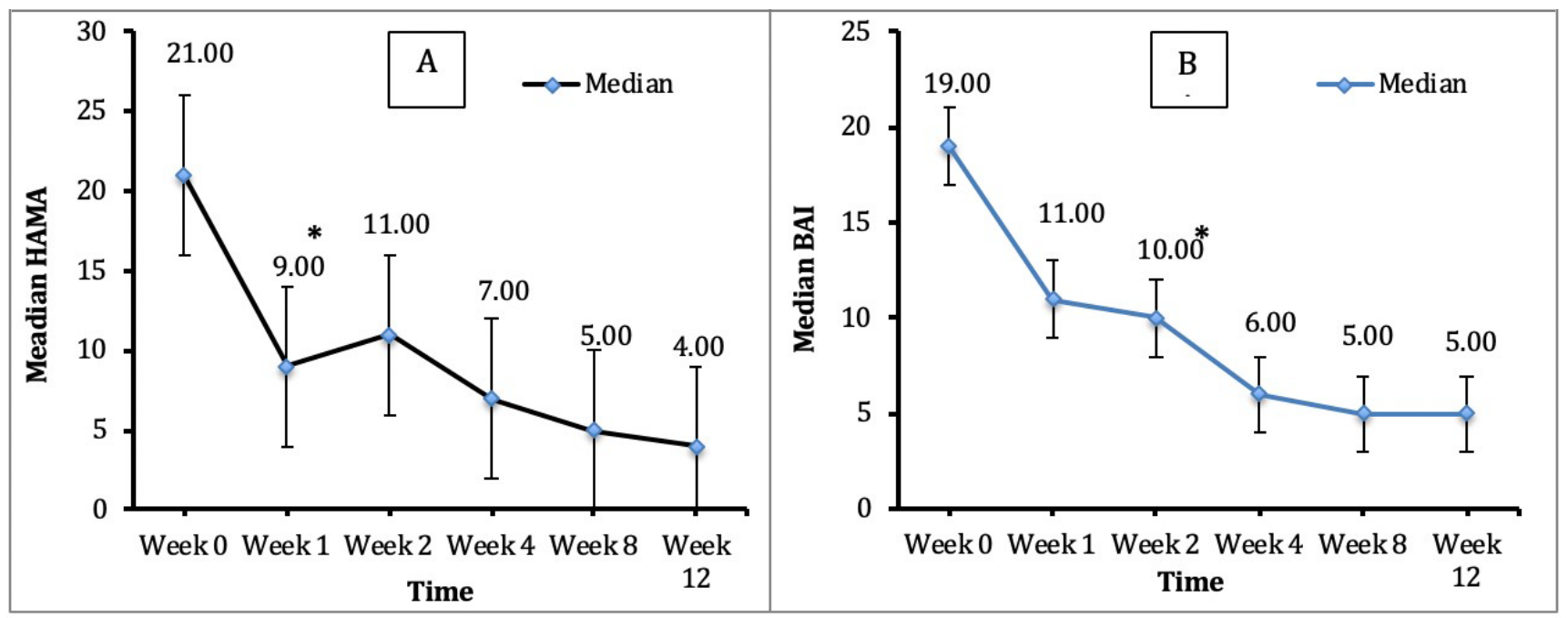

Figure 4: Changes in HAMA and BAI scores from baseline through Week 12. A) Change in Median HAMA score; B) Change in Median BAl score. Note: ${ }^{*} P<0.05$ compared to baseline.

HAMA: Hamilton Rating Scale for Anxiety; BAI: B.

Figure 3B, Figure $3 \mathrm{C}$ and Figure $3 \mathrm{D}$ ) and in HAMA score (Figure 4A) (all p < 0.001). Only the significant fall in BAI score reached statistical significance by the $2^{\text {nd }}$ week ( $p$
$=0.011$ ) (Figure 4B).

When the patient group was divided into a high and 


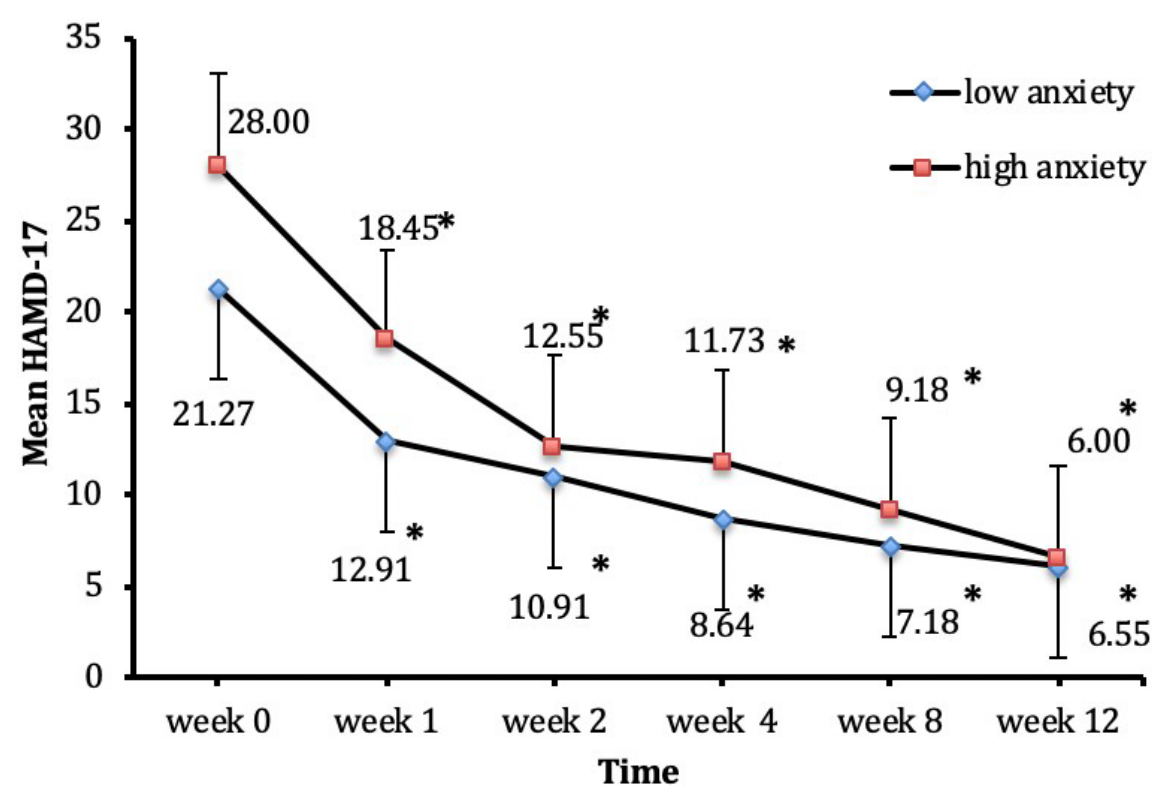

Figure 5: Decrease of HAMD-17 score over time in high and low anxiety patients. There was a significant change in HAMD17 score over time across the whole sample $F(2.87,57.93)=35.58, p=0.0005$ but changes in HAMD-17 over time were equivalent across the two groups $F(1.20)=1.52, p=0.232$.

Note: ${ }^{*} P<0.05$ compared to baseline.

HAMD: Hamilton Rating Scale for Depression.

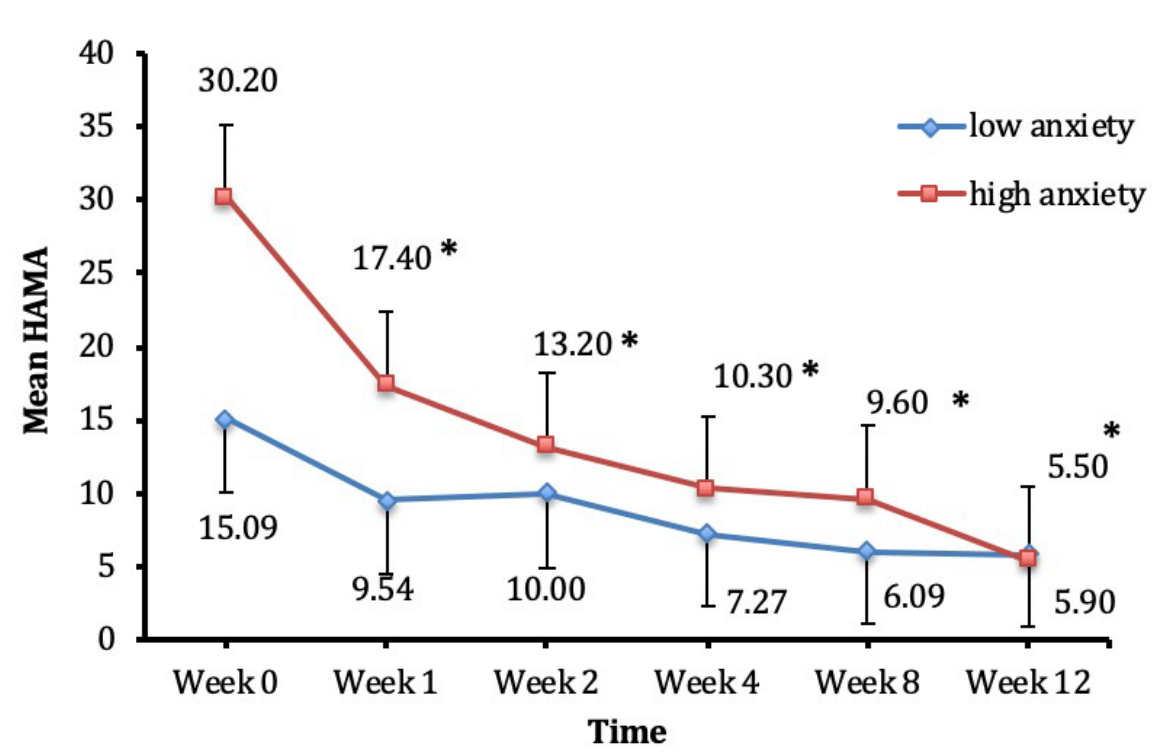

Figure 6: Decrease of HAMA score over time in high and low anxiety patients. There was a significant effect of time across six time points $F(2.75,52.26)=28.84, p=0.0005$ more so for the high anxiety group; and a borderline significant difference between groups $F(1.19)=3.72, p=0.006$ and a significant interaction between time and group $F(2.75,52.26)=5.99, p=$ 0.002 in HAMA score.

Note: ${ }^{*} P<0.05$ compared to baseline.

HAMA: Hamilton Rating Scale for Anxiety.

a low anxiety group by taking a median split of HAMA of 22, mixed ANOVA analysis revealed significant changes in HAMD-17 score over time across the whole sample $F$ $(2.87,57.93=35.58, p=0.0005$ but changes in HAMD17 over time were equivalent across the two groups $F$ $(1.20)=1.52, p=0.232$. There was no significant interaction between time and group $F(2.87,57.93)=1.16$, $p=0.330$ and follow up of interaction indicated no significant difference between groups at baseline and the mean scores of both groups decreased similarly over time (Figure 5). Mixed ANOVA analysis for HAMA scores on the other hand, revealed a significant difference across six time points $F(2.75,52.26)=28.84, p=$ 0.0005 ; a significant difference between groups $F(1.19)$ $=3.72, p=0.006$; and a significant interaction between time and group $F(2.75,52.26)=5.99, p=0.002$ in HAMA score. Pursuing this interaction further, we determined that, although there was a significant difference between the two groups at baseline, the mean HAMA score decrease over time was far more pronounced for those 
in the high anxiety group and there was no difference in the level of anxiety by week 2 between the two groups (Figure 6).

Table 3: Side effect frequency

\begin{tabular}{|c|c|c|}
\hline Side effect & $\begin{array}{l}\text { QXR } \\
(N=39)\end{array}$ & Frequency \\
\hline Fatigue & 6 & $15.4 \%$ \\
\hline Insomnia & 3 & $7.7 \%$ \\
\hline Drowsiness & 10 & $25.6 \%$ \\
\hline Dry mouth & 13 & $33.3 \%$ \\
\hline Nausea & 1 & $2.6 \%$ \\
\hline GI-constipation & 2 & $5.1 \%$ \\
\hline Dizziness & 1 & $2.6 \%$ \\
\hline Headache & 1 & $2.6 \%$ \\
\hline Weight gain & 5 & $12.8 \%$ \\
\hline Increased appetite & 5 & $12.8 \%$ \\
\hline Increased irritability & 5 & $12.8 \%$ \\
\hline Diarrhea & 2 & $5.1 \%$ \\
\hline Decreased libido & 1 & $2.6 \%$ \\
\hline Morning grogginess & 15 & $38.5 \%$ \\
\hline Nightmares & 1 & $2.6 \%$ \\
\hline Increased anxiety & 7 & $17.9 \%$ \\
\hline Flatulence & 1 & $2.6 \%$ \\
\hline Double/Blurred vision & 2 & $5.13 \%$ \\
\hline Palpitations & 1 & $2.6 \%$ \\
\hline Chest pain & 1 & $2.6 \%$ \\
\hline Amotivation & 1 & $2.6 \%$ \\
\hline Orthostatic hypotension & 1 & $2.6 \%$ \\
\hline Urinary urgency & 1 & $2.6 \%$ \\
\hline Psychomotor retardation & 2 & $5.1 \%$ \\
\hline Sweating & 1 & $2.6 \%$ \\
\hline Increased muscle tension & 1 & $2.6 \%$ \\
\hline Decreased appetite & 1 & $2.6 \%$ \\
\hline Joint stiffness & 1 & $2.6 \%$ \\
\hline No reported side effects & 5 & $12.8 \%$ \\
\hline $\begin{array}{l}\text { Patients who dropped out due to } \\
\text { side effects of dry mouth, fatigue, } \\
\text { irritability }\end{array}$ & 4 & $10.3 \%$ \\
\hline
\end{tabular}

QXR: Quetiapine Extended Release.

\section{Quetiapine dosing and tolerability}

In terms of dosing, our mean effective dose at week 8 was $176.9 \mathrm{mg} / \mathrm{d}$ and the mean Quetiapine blood level was $5.07 \pm 7.68 \mathrm{ng} / \mathrm{ml}$. Of the 43 patients who were included in our ITT group, 10 patients discontinued treatment due to medication side effects. Patients reported side effects in the following categories: sedative, cardiovascular, anticholinergic, gastrointestinal, vegetative, autonomic, neurological, sexual and edema-related side effects. As seen in Table 3, the most consistently reported side effects were morning grogginess (35.8\%), dry mouth (33.3\%) and drowsiness (25.6\%). Four patients dropped out of the study due to dry mouth, fatigue, and increased irritability. In terms of longitudinal side effects over the entire study, dizziness, dry mouth, nausea, upset stomach, sweating, flushing and headache were reported fairly consistently throughout the 12 weeks of the study.

In terms of metabolic side effects, although patients did report increased appetite and weight gain, our physical assessments showed no significant change in weight (91.3 vs. 93.3) or BMI (32.1 vs. 32.3) after 12 weeks of treatment $(p=0.372)$. As seen in Table 4 and Figure 7 , our results showed no significant increase in risk factors for metabolic syndrome (e.g. total cholesterol, triglycerides, HDL, LDL, and glucose).

\section{Discussion}

Quetiapine XR was originally introduced as an atypical antipsychotic and is currently Food and Drug Administration (FDA)-approved for the treatment of 1) Schizophrenia; 2) Depressive, manic, or mixed episodes in Bipolar I disorder. The main mechanism of action of Quetiapine as an antipsychotic involves its core receptor activities as a dopamine receptor-2 (D2) antagonist, and as a more potent serotonin receptor- $2 \mathrm{~A}\left(5 \mathrm{HT}_{2 \mathrm{~A}}\right)$ antagonist and/or $5 \mathrm{HT}_{1 \mathrm{~A}}$ agonist [16]. However, as of 2013, Quetiapine was also shown to exert some antidepressant effects and was approved as an adjunctive therapy for patients with MDD who were refractory to standard antidepressant monotherapies (i.e. amitriptyline, bupropion, citalopram, duloxetine, escitalopram, fluoxetine, paroxetine, sertraline or venlafaxine) [17]. It is believed that Quetiapine's major metabolite, norquetiapine,

Table 4: Metabolic syndrome variables

\begin{tabular}{|l|l|l|l|l|}
\hline & Pre-treatment (Baseline) & Post-treatment (Week 12) & t/z & p \\
\hline Cholesterol & $190.80(46.34)$ & $193.54(42.77)$ & $-0.874^{\mathrm{t}}$ & 0.389 \\
\hline Triglycerides & $87(155)$ & $86(176)$ & $-0.625^{z}$ & 0.532 \\
\hline HDL & $49.71(15.62)$ & $48.66(14.88)$ & $1.170^{\mathrm{t}}$ & 0.250 \\
\hline LDL & $124.32(80.61)$ & $127.32(40.21)$ & $-1.089^{\mathrm{t}}$ & 0.284 \\
\hline Glucose & $93.18(11.40)$ & $93.87(13.99)$ & $-0.434^{\mathrm{t}}$ & 0.667 \\
\hline
\end{tabular}

For continuous variables, mean (standard deviation) or median (interquartile range) is given according to the test applied and $\mathrm{t}$ scores are obtained for paired t test, $z$ scores are obtained for two related samples test.

Note: ${ }^{*} p<0.05$. 


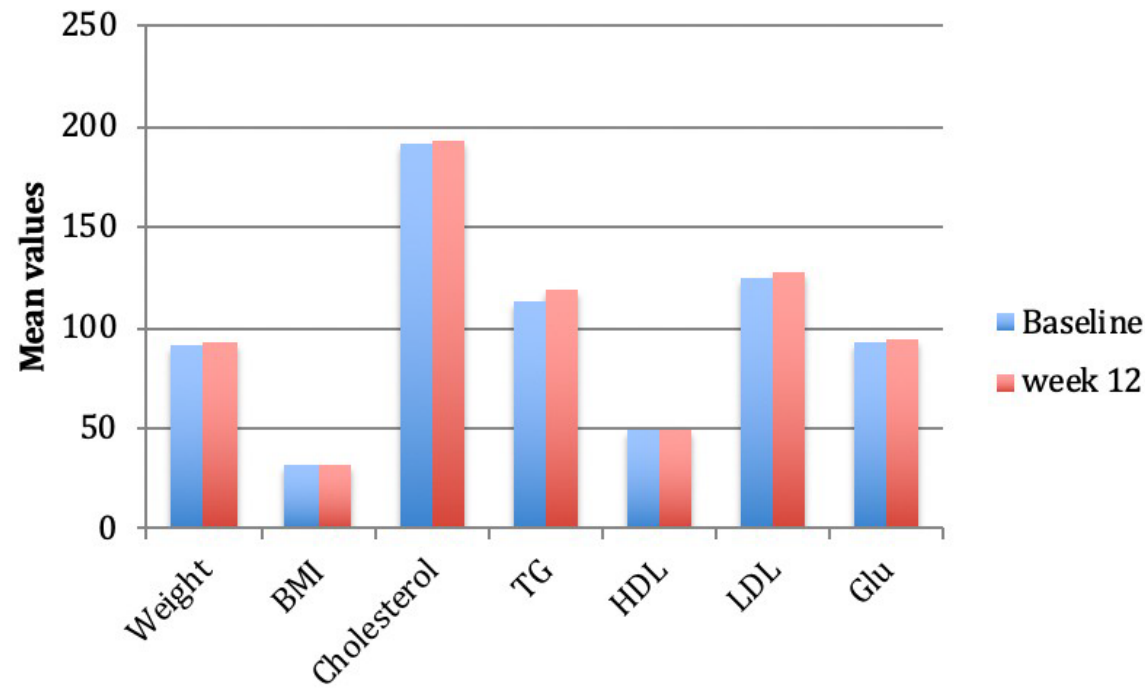

Figure 7: Metabolic syndrome variables

BMI: Body Mass Index; TG: Triglyceride; HDL: High Density Lipoprotein; LDL: Low Density Lipoprotein; Glu: Glucose

is the likely explanation for this antidepressant effect. Norquetiapine has a high affinity blockade for the Norepinephrine Transporter (NET) and also has partial agonistic effects on the $5 \mathrm{HT}_{1 \mathrm{~A}}$ receptor $[18,19]$.

Since 2009, five studies have tested and proved the efficacy and tolerability of Quetiapine XR as monotherapy for MDD [20-24]. Quetiapine XR was specifically reported as an effective treatment in elderly patients (i.e. mean age 71.3 years) in a study using flexible dosing Quetiapine 60-300 mg/day [24]. Improvement in depression symptoms as early as week 1 was reported in three other studies of 6 and 10-weeks duration [2022]. A $66 \%$ decrease in recurrence of depressive episodes $(p<0.001)$ was reported in patients stabilized and then maintained on Quetiapine XR up to 52 weeks in another study [23].

The results of our 12-week study both confirm and expand the published reports on Quetiapine XR as effective and well tolerated monotherapy for the treatment of MDD [20-24]. Our results clearly demonstrate the efficacy of Quetiapine XR in reducing depressive symptoms as early as week 1 . Likewise, we saw a significant decrease in anxiety symptoms as well based on our HAMA data. The decrease in anxiety was much more pronounced in the high anxiety group and the reduction in anxiety levels was similar by week 2 in both groups. HAMD-17 scores on the other hand, decreased similarly in both high and low anxiety groups. From a pooled analysis of two previous randomized, double blind, placebo-controlled studies of Quetiapine XR monotherapy studies, a significant reduction in Montgomery Asberg Depression Rating Scale (MADRS) total score was found in patients with high and lower levels of anxiety (anxious depression defined as baseline HAM-A total score of $\geq 20$ ) with Quetiapine XR 150 and $300 \mathrm{mg} /$ day [25]. In elderly MDD patients, a significant reduction in MADRS total score was seen compared with placebo in the higher anxiety and lower anxiety subgroups by week 9 with Quetiapine XR (50-300 mg/day) monotherapy [26]. These findings showed that Quetiapine monotherapy was effective in relieving depressive symptoms in patients with MDD, irrespective of baseline levels of anxiety. It is generally accepted that comorbid anxiety with depression usually predicts poor outcomes with a higher percentage of treatment resistance than either disorder occurring alone [27]. Therefore, Quetiapine XR monotherapy may be a pharmacological option for anxious depression. Support for this conclusion is provided by a fMRI study. In anxious MDD patients increased task-induced left amygdala activation, decreased resting state amygdala-cortical and amygdala-pons connectivity were established as compared to healthy controls. After treatment with Quetiapine increased amygdala-cortical connectivity was detected at week 2 of treatment, which was maintained at week 8 [28]. This finding is corroborated by the steep decrease in HAMA scores by week 2 in our group of high anxiety patients. In the above referenced study, additional areas that showed increased connectivity included insula and putamen in anxious MDD patients at week 8 , and the decrease in HAMA scores correlated with an increase in amygdala-cuneus and parietal cortex connectivity [28]. These results indicate that Quetiapine XR treatment of anxious MDD patients is associated with modulation of amygdala connectivity. This increased connectivity may be due to the neurotrophic effect of Quetiapine and its metabolite, norquetiapine. This assumption is based on a molecular cell culture study, in which Quetiapine and norquetiapine induced ERK/ MAPK (extracellular signal regulated kinase/mitogen protein activated kinase) signaling and caused a consequent GDNF (glia cell line derived neurotrophic factor) release at the lowest drug concentration in a 
dose-dependent manner similar to the activation pattern of the antidepressant, reboxetine. Early peak of ERK activation was observed at 10 minutes and was sufficient to induce long term GDNF release 48 hours after norquetiapine and reboxetine treatment which suggests initiation of the cascade of adaptive changes responsible for clinical effects after three-four weeks or sooner after the beginning of treatment [29].

Stress perception also decreased significantly in our study. Early life stress plays an important role in the onset of depression and is associated with poor response to treatment. Furthermore, it triggers epigenetic changes associated with the pathophysiology of MDD. In an animal model of depression, maternally deprived rats showed depressive-like behavior in the forced swim test and they had an increase in the histone deacetylase (HDAC) and DNA methyltransferase (DNMT) enzymatic activities in the hippocampus and nucleus accumbens, while treatment with Quetiapine reversed depressive-like behavior and the DNMT activity in the hippocampus [30]. Although we did not measure childhood trauma, stress in the month preceding enrollment in our study due to depression may have also exerted epigenetic changes and these changes may have been reversed by Quetiapine treatment.

With respect to efficacy and tolerability, we found that the most effective average daily dose with the least amount of intolerable side effects was around $175 \mathrm{mg}$. Since NET inhibition is accepted as a mechanism of antidepressant activity of Quetiapine, NET occupancy was measured via positron emission tomography in another study; NET occupancy in the thalamus was found to be 19 and $35 \%$, respectively, at Quetiapine XR doses of 150 and $300 \mathrm{mg} / \mathrm{d}$ and the estimated plasma concentration of norquetiapine corresponding to $50 \%$ NET occupancy was $161 \mathrm{ng} / \mathrm{ml}$ [31]. Although we did not measure plasma concentration of norquetiapine, we measured Quetiapine plasma concentration and it was $5.07 \mathrm{ng} / \mathrm{ml}$ by week 8 and our dose range was $25-300 \mathrm{mg} / \mathrm{d}$. Nevertheless, the precise dosing of Quetiapine XR as monotherapy for MDD remains as an open question. It is believed that the antidepressant activity becomes manifest at lower doses than the dose ranges prescribed for psychotic symptoms, which is usually $>300 \mathrm{mg} /$ day. In one study, Quetiapine XR $150 \mathrm{mg} /$ day was consistently more efficacious than $50 \mathrm{mg} /$ day and 300 $\mathrm{mg} /$ day [21] while in another study, remission rates (MADRS score $\leq 8$ ) were significantly higher for Quetiapine XR $300 \mathrm{mg} /$ day, but not for Quetiapine XR $150 \mathrm{mg} /$ day [20]. Most recently, a retrospective study demonstrated that the mean effective initial and maintenance doses for Quetiapine as an augmentation treatment for depression were 23.6 to $40.7 \mathrm{mg}$ / day, respectively [32]. A post hoc analysis of pooled data from four previously reported randomized, placebo-controlled studies of Quetiapine XR 150 and
$300 \mathrm{mg} /$ day, as monotherapy, or adjunctive therapy to ongoing antidepressant therapy showed that the proportion of patients experiencing sustained response was greater with quetiapine XR $150 \mathrm{mg} /$ day versus placebo at week 2 in the monotherapy studies [33]. This conflicting data on proper dosage requires further study, especially with regard to the drug's tolerability.

In terms of tolerability, the reported side effect profile for Quetiapine XR at both the $150 \mathrm{mg} /$ day and $300 \mathrm{mg} /$ day dosing predominately includes somnolence, sedation and dry mouth. In one of the randomized, double-blind, placebo-controlled studies, sedation was found as the most common side effect leading to discontinuation [21]. In a meta-analysis of randomized, placebo-controlled trials of Quetiapine monotherapy in MDD, the pooled mean change in Pittsburgh Sleep Quality Index scores of the quetiapine-treated group was found to be significantly higher than that of the placebo-treated group. ${ }^{7}$ In another study, $19.7 \%$ of patients discontinued Quetiapine XR $150 \mathrm{mg} /$ day and $15.1 \%$ discontinued Quetiapine XR $300 \mathrm{mg} /$ day due to dry mouth, sedation, and somnolence [20]. Quetiapine studies have shown that the tolerability is related to dosing and a lower dose is better tolerated than a higher dose in the treatment of depression [34]. In our study, the discontinuation rate due to side effects was $23 \%$ and the most prominent side effects were extended sleep, early morning grogginess and dry mouth. The blockade of histamine 1 receptors and alpha1 receptors may play a major role in antipsychotic drug-induced sedation [35]. Because of sedation, in our study the starting dose was decreased from $50 \mathrm{mg}$ to $25 \mathrm{mg} / \mathrm{d}$ and the up-titration was done more slowly. These results are consistent with patient reports in the other studies of Quetiapine XR in this dosing range [20-24] and indicate that the actual doses of Quetiapine in MDD should be much lower than the doses used in placebo-controlled clinical trials and the dose titration should start as low as $25 \mathrm{mg} / \mathrm{d}$ and increased slowly as tolerated to prevent non-compliance.

Metabolic syndrome is another important side effect to consider for second generation antipsychotics including Quetiapine XR [34,36]. Hyperglycemia, hyperlipidemia, weight gain, and new-onset diabetes have all been reported in patients taking standard doses of Quetiapine $[35,37]$. In fact, current manufacturer guidelines for Quetiapine Fumarate (Seroquel ${ }^{\circledR}$ ) recommend monitoring plasma glucose and lipid profiles in all patients with significant risk factors (e.g. obesity) (AstraZeneca Pharmaceuticals, London, United Kingdom) [36,38]. But, our study participants showed no significant signs of increased risk factors for Metabolic Syndrome with a mean dose of $175 \mathrm{mg}$ during the 12-week observation period.

In conclusion, Quetiapine XR monotherapy is both efficacious and tolerable in the treatment of MDD with symptom improvement seen as early as week 1 and the optimal dose being between 150-175 mg/day. Early 
symptom improvement might be a predictor of response to Quetiapine treatment, a finding that could give doctors confidence to continue treatment and may facilitate adherence to treatment [6].

Limitations of this study are the small number of completers. Loss to attrition, absence of a placebo-controlled, double blind, randomized arm and a 12-week treatment phase with no measurement of norquetiapine blood levels. Further research is needed to determine the long-lasting impact of Quetiapine XR in the treatment of $\mathrm{MDD}$, and long-term remission rates, and long-term side effect profile.

\section{Statement of Conflict of Interest}

This study was funded by an Investigator-initiated grant awarded to Dr. Angelos Halaris by AstraZeneca, the manufacturer of Seroquel (Quetiapine Fumarate).

\section{Conflicts of Interest and Source of Funding}

Authors have none to declare. This study was funded with a grant from AstraZeneca.

\section{References}

1. Depression Fact Sheet. World Health Organization.

2. The Global Burden of Disease: 2004 Update. World Health Organization.

3. Greenberg PE, Fournier AA, Sisitsky T, Pike CT, Kessler RC (2015) The economic burden of adults with major depressive disorder in the United States (2005 and 2010). J Clin Psychiatry 76: 155-162.

4. Wang G, Mclntyre A, Earley WR, Raines SR, Eriksson H (2014) A randomized, double-blind study of the efficacy and tolerability of extended release quetiapine fumarate (quetiapine XR) monotherapy in patients with major depressive disorder. Neuropsychiatr Dis Treat 10: 201-216.

5. Neutel JM, Smith DH, Weber MA (2011) Low-dose combination therapy: An important first-line treatment in the management of hypertension. Am J Hypertens 14: 286-292.

6. McIntyre RS, Gorwood P, Thase ME, Liss C, Desai D, et al. (2015) Early symptom improvement as a predictor of response to extended release quetiapine in major depressive disorder. J Clin Psychopharmacol 35: 706-710.

7. Maneeton N, Maneeton B, Srisurapanont M, Martin SD (2012) Quetiapine monotherapy in acute phase for major depressive disorder: A meta-analysis of randomized, placebo-controlled trials. BMC Psychiatry 12: 160.

8. Culpepper L (2011) Understanding the burden of depression. J Clin Psychiatry 72: 19.

9. Rihmer Z (2009) Antidepressive efficacy of quetiapine $X R$ in unipolar major depression-the role of early onset of action and sleep-improving effect in decreasing suicide risk. Neuropsychopharmacol Hung 11: 211-215.

10. American Psychiatric Association (2010) Practice guideline for the treatment of patients with major depressive disorder. ( $3^{\text {rd }}$ Edn), APA Publishing, Washington, USA.

11. Masand PS (2003) Tolerability and adherence issues in antidepressant therapy. Clin Ther 25: 2289-2304.

12. Cassano P, Fava M (2004) Tolerability issues during longterm treatment with antidepressants. Ann Clin Psychiatry 16: 15-25.
13. Global Database on Body Mass Index. World Health Organization.

14. Hamilton M (1960) A rating scale for depression. J Neurol Neurosurg Psychiatry 23: 56-62.

15. Maier W, Buller R, Philipp M, Heuser I (1988) The Hamilton Anxiety Scale: Reliability, validity and sensitivity to change in anxiety and depressive disorders. J Affect Disord 14: 6168.

16. Díaz-Mataix L, Scorza MC, Bortolozzi A, Toth $M$, Celada $P$, et al. (2005) Involvement of 5-HT1A receptors in prefrontal cortex in the modulation of dopaminergic activity: Role in atypical antipsychotic action. J Neurosci 25: 10831-10843.

17. Pae CU, Sohi MS, Seo HJ, Serretti A, Patkar AA, et al. (2010) Quetiapine XR: Current status for the treatment of major depressive disorder. Prog Neuropsychopharmacol Biol Psychiatry 34: 1165-1173.

18. Jensen NH, Rodriguiz RM, Caron MG, Wetsel WC, Rothman RB, et al. (2008) N-Desalkylquetiapine, a Potent Norepinephrine Reuptake Inhibitor and Partial 5-HT(1A) Agonist, as a Putative Mediator of Quetiapine's Antidepressant Activity. Neuropsychopharmacology 33: 2303-2312.

19. Prieto E, Mico JA, Meana JJ, Majadas S (2010) Neurobiological bases of quetiapine antidepresant effect in the bipolar disorder. Actas Esp Psiquiatr 38: 22-32.

20. Cutler AJ, Montgomery SA, Feifel D, Lazarus A, Aström $M$, et al. (2009) Extended release quetiapine fumarate monotherapy in major depressive disorder: A placebo-and duloxetine-controlled study. J Clin Psychiatry 70: 526-539.

21. Weisler R, Joyce M, McGill L, Lazarus A, Szamosi J, et al. (2009) Extended release quetiapine fumarate monotherapy for major depressive disorder: Results of a double-blind, randomized, placebo-controlled study. CNS Spectr 14: 299-313.

22. Bortnick B, El-Khalili N, Banov M, Adson D, Datto C, et al. (2011) Efficacy and tolerability of extended release quetiapine fumarate (quetiapine XR) monotherapy in major depressive disorder: A placebo-controlled, randomized study. J Affect Disord 128: 83-94.

23. Liebowitz M, Lam RW, Lepola U, Datto C, Sweitzer D, et al. (2010) Efficacy and tolerability of extended release quetiapine fumarate monotherapy as maintenance treatment of major depressive disorder: A randomized, placebo-controlled trial. Depress Anxiety 27: 964-976.

24. Katila H, Mezhebovsky I, Mulroy A, Berggren L, Eriksson H, et al. (2013) Randomized, double-blind study of the efficacy and tolerability of extended release quetiapine fumarate (quetiapine XR) monotherapy in elderly patients with major depressive disorder. Am J Geriatr Psychiatry 21: 769-784.

25. Thase ME, Demyttenaere K, Earley WR, Gustafsson U, Udd M, et al. (2012) Extended release quetiapine fumarate in major depressive disorder: Analysis in patients with anxious depression. Depress Anxiety 29: 574-586.

26. Montgomery SA, Altamura AC, Katila H, Datto C, Szamosi $J$, et al. (2014) Efficacy of extended release quetiapine fumarate monotherapy in elderly patients with major depressive disorder: Secondary analyses in subgroups of patients according to baseline anxiety, sleep disturbance, and pain levels. Int Clin Psychopharmacol 29: 93-105.

27. Coplan JD, Aaronson CJ, Panthangi V, Kim Y (2015) Treating comorbid anxiety and depression: Psychosocial and pharmacological approaches. World J Psychiatry 5: 366378.

28. Altinay M, Karne H, Beall E, Anand A (2016) Quetiapine 
extended release open-label treatment associated changes in amygdala activation and connectivity in anxious depression: An fMRI Study. J Clin Psychopharmacol 36: 562-571.

29. Di Benedetto B, Kühn R, Nothdurfter C, Rein T, Wurst W, et al. (2012) N-desalkylquetiapine activates ERK1/2 to induce GDNF release in $\mathrm{C} 6$ glioma cells: A putative cellular mechanism for quetiapine as antidepressant. Neuropharmacology 62: 209-216.

30. Ignácio ZM, Réus GZ, Abelaira HM, Maciel AL, de Moura $A B$, et al. (2017) Quetiapine treatment reverses depressive-like behavior and reduces DNA methyltransferase activity induced by maternal deprivation. Behav Brain Res 320: 225-232.

31. Nyberg $S$, Jucaite A, Takano A, Kågedal M, Cselényi Z, et al. (2013) Norepinephrine transporter occupancy in the human brain after oral administration of quetiapine XR. Int J Neuropsychopharmacol 16: 2235-2244.

32. Pae CU, Wang SM, Han C, Lee SJ, Patkar AA, et al. (2015) Quetiapine augmentation for depression: Dosing pattern in routine practice. Int Clin Psychopharmacol 30: 54-58.

33. Vieta E, Bauer M, Montgomery S, Mclntyre RS, Szamosi J, et al. (2013) Pooled analysis of sustained response rates for extended release quetiapine fumarate as monotherapy or adjunct to antidepressant therapy in patients with major depressive disorder. J Affect Disord 150: 639-643.

34. Gao K, Kemp DE, Fein E, Wang Z, Fang Y, et al. (2011) Number needed to treat to harm for discontinuation due to adverse events in the treatment of bipolar depression, major depressive disorder, and generalized anxiety disorder with atypical antipsychotics. J Clin Psychiatry 72: 10631071.

35. Fang F, Sun H, Wang Z, Ren M, Calabrese JR, et al. (2016) Antipsychotic drug-induced somnolence: Incidence, mechanisms, and management. CNS Drugs 30: 845-867.

36. Pramyothin P, Khaodhiar L (2010) Metabolic syndrome with the atypical antipsychotics. Curr Opin Endocrinol Diabetes Obes 17: 460-466.

37. Jin H, Meyer JM, Jeste DV (2004) Atypical antipsychotics and glucose dysregulation: A systematic review. Schizophr Res 71: 195-212.

38. Seroquel XR (2019) Summary of product characteristics, labelling and package leaflet. 